\title{
OPEN Propionic acid produced by Cutibacterium acnes fermentation ameliorates ultraviolet B-induced melanin synthesis
}

\author{
Hsin-Jou Kao ${ }^{1}$, Yan-Han Wang ${ }^{2}$, Sunita Keshari ${ }^{3}$, John Jackson Yang ${ }^{3}$, Shinta Simbolon ${ }^{1}$, \\ Chun-Chuan Chen ${ }^{1}$ \& Chun-Ming Huang ${ }^{1 凶}$
}

Ultraviolet irradiation induces melanin accumulation, which can be reduced by the use of chemical whitening products. However, the associated safety concerns of such products have prompted the search for natural and harmless alternatives. This study aimed to identify a natural acidic formulation to reduce skin pigmentation. The metabolite propionic acid $\left(\mathrm{CH}_{3} \mathrm{CH}_{2} \mathrm{COOH}, \mathrm{PA}\right)$ was the most abundant fatty acid in the filtrate from Pluronic F68 (PF68) fermentation of Cutibacterium acnes ( $C$. acnes) and reduced the DOPA-positive melanocytes by significantly inhibiting cellular tyrosinase activity via binding to the free fatty acid receptor 2 (FFAR2). Moreover, $4 \mathrm{mM}$ PA treatment did not alter melanocyte proliferation, indicating that it is an effective solution for hyperpigmentation, causing no cellular damage. The reduced DOPA-positive melanocytes and tyrosinase activity were also observed in mice ear skin tissue injected with a mixture of $C$. acnes and PF68, supporting that the inhibition of melanogenesis is likely to be mediated through fermentation metabolites from C. acnes fermentation using PF68 as a carbon source. Additionally, PA did not affect the growth of its parent bacteria $C$. acnes, hence is a potent fermentation metabolite that does not disrupt the balance of the skin microbiome.

Skin acts as an interface between the human body and the external environment providing defense against pathogens, physical and ultraviolet damage ${ }^{1}$. If human skin is over exposed to ultraviolet radiation (UVR) that influences the function and survival of many cell types, inducing skin inflammation, which can lead to cancer ${ }^{2,3}$. UV-induced DNA damage activates cellular repair signals to produce melanin in melanocytes, resulting in skin pigmentation ${ }^{4,5}$. The healthy human skin surface is colonized by a diverse milieu of microorganisms, many of which are harmless as commensals or opportunistic pathogens ${ }^{6,7}$. Probiotics are common examples of bacteriotherapy with the potential for photoprotection by slowing the signs of aging skin and mitigating the effects of UV-induced skin inflammation ${ }^{3,8,9}$. For example, probiotic lactic acid bacteria notably prevents UV-induced inflammatory, allergic reactions, and immunosuppression in the $\operatorname{skin}^{10}$. Moreover, the ability of Cutibacterium acnes (C. acnes), a predominant bacterium in the skin microbiome, to produce porphyrin in response to UVR makes it a major biomarker owing to its role in protecting and preventing disequilibrium ${ }^{2,11,12}$, hence it is of practical interest ${ }^{13}$. Studies revealed that galactomyces ferment filtrate (GFF) reduced melanin in human melanoma cells, thereby reducing skin pigmentation ${ }^{14}$. Free fatty acids (FFA) have remarkable regulatory effects on melanogenesis and suppressed tyrosinase activity in cultured B16F10 murine melanoma cells ${ }^{15}$. Most treatment options for hyperpigmentation block the conversion of tyrosine to melanin, acting as a tyrosinase inhibitor, a key regulatory enzyme required for melanin biosynthesis ${ }^{16}$. Recently, the extensive use of skin whitening agents, like phenolic and mercury compounds, in cosmetic formulations have raised serious safety concerns ${ }^{17}$. Moreover, FFAR2 (also known as GPR43) is present in a variety of tissues like bone marrow, spleen, and normal skin ${ }^{18}$ and is a promising drug targets for obesity, colitis, and some inflammatory responses ${ }^{19}$. FFAR2 is a receptor for

${ }^{1}$ Department of Biomedical Sciences and Engineering, National Central University, Zhongda Rd, No. 300, Zhongda Rd., Zhongli District, Taoyuan City 32001, Taiwan, ROC. '2Department of Dermatology, University of California, San Diego, CA, USA. ${ }^{3}$ Department of Life Sciences, National Central University, Zhongli District, Taoyuan City, Taiwan, ROC. ${ }^{\boxplus}$ email: chunmingsd@gmail.com 
short-chain fatty acids (SCFAs) with chain lengths less than six carbons such as acetate, butyrate, and propionate, the most potent agonist for FFAR2 ${ }^{20,21}$. Previously, we demonstrated that in vivo knockdown of FFAR2 in mouse skin blocked butyric acid mediation of UV irritation ${ }^{3}$.

C. acnes is abundant on the human skin surface accounting for $>60 \%$ of the bacteria ${ }^{2}$. Moreover, propionic acid (PA), a fermentation metabolite from C. acnes, and its esterified derivatives, act as a potential antimicrobial agent against Staphylococcus aureu $u^{11,22}$ and poly(ethylene oxide) coatings are a promising method to avoid infections ${ }^{23}$. Skin commensal probiotic bacteria can inhibit the growth of USA300 through fermentation of poly(ethylene glycol) dimethacrylate as a selective fermentation initiator ${ }^{24}$. PF68, PEG-based polymer is a stable gel carrier for antimicrobial agents, increasing the surface solubility of the drug, and is usually used in the treatment of infected wounds without any discernible side effects ${ }^{25}$. In this study, we determined that PF68 as a polymer derived compound is a potentially safe and effective agent and the application of metabolites from the fermentation of PF68 by the skin commensal C. acnes could inhibit UV-induced hyperpigmentation or melanogenesis of skin.

\section{Methods}

Ethics statement. This study was carried out in strict with an approved Institutional Animal Care and Use Committee (IACUC) protocol at National Central University (NCU), Taiwan (NCU-106-016) and in compliance with the Arrive guidelines (https://arriveguidelines.org/). Institute Cancer Research (ICR) mice (8-9 weeks old females; National Laboratory Animal Center, Taipei, Taiwan) were sacrificed using $\mathrm{CO}_{2}$ in a closed box. All methods were performed in accordance with relevant guidelines and regulations.

Bacterial culture. C. acnes (ATCC 6919) was cultured on Reinforced Clostridium Medium (RCM, Oxford, Hampshire, England) under anaerobic conditions using a Gas-Pak (BD, Sparks, MD, USA). Bacteria were cultured at $37^{\circ} \mathrm{C}$ until the logarithmic growth phase. Bacterial pellets were harvested by centrifugation at $5,000 \times \mathrm{g}$ for $10 \mathrm{~min}$, washed in phosphate-buffered saline (PBS), and then suspended in PBS or RCM for further experiments.

Bacteria fermentation. C. acnes $\left(10^{5}\right.$ colony-forming unit $\left.(\mathrm{CFU}) / \mathrm{mL}\right)$ was incubated in $10 \mathrm{~mL}$ TSB in the presence or absence of $2 \%$ PF68 (BASF, NY, USA) under anaerobic conditions using Gas-Pak at $37^{\circ} \mathrm{C}$. PF68 alone in TSB was included as a control. The $0.002 \%(\mathrm{w} / \mathrm{v})$ phenol red (Sigma) in TSB served as a fermentation indicator. A color change from red-orange to yellow indicated the occurrence of bacterial fermentation, which was detected by optical density at $560 \mathrm{~nm}\left(\mathrm{OD}_{560}\right)$.

Gas chromatography-mass spectrometry (GC-MS) analysis. C. acnes $\left(10^{5} \mathrm{CFU} / \mathrm{mL}\right)$ was incubated in TSB $(10 \mathrm{~mL})$ in the presence of $2 \%$ PF68. After 1 day fermented media was centrifuged at $5000 \mathrm{~g}$ for $10 \mathrm{~min}$ and the supernatant was used for detection of SCFAs using previously published protocol ${ }^{26}$. The levels of acetic acid (AA), PA, butyric acid (BA), and iso-butyric acid (I-BA) in the fermentation media were quantified by a calibration curve made from six non-zero levels using the FFA Test Standard (Restek Corporation, Bellefonte, PA, USA) which is diluted to 500-, 1,000-, 2,000-, 5,000- and 10,000-fold.

B16F10 melanoma cell culture. B16F10 melanoma cell line was kindly provided by Dr. Richard Gallo, University of California San Diego, USA, and Dr. Cheng Ching-Yi, Chang Gung University of Science and Technology, Taiwan. The cells were cultured in Dulbecco's Modified Eagle's Medium (DMEM, Life Technologies, Carlsbad, CA, USA) supplemented with 10\% fetal bovine serum (FBS, Life Technologies) and 1\% Penicillinstreptomycin (Life Technologies) at $37^{\circ} \mathrm{C}$ and $5 \% \mathrm{CO}_{2}$. The cells were grown until $70-80 \%$ confluence and then subcultured with Trypsin-Ethylenediaminetetraacetic acid (EDTA, Life Technologies).

Reverse transcription-quantitative polymerase chain reaction (RT-qPCR). RT-qPCR was employed to study the tyrosinase gene expression in B16F10 melanoma cells treated with $4 \mathrm{mM}$ PA for $48 \mathrm{~h}$. Total cellular RNA was extracted using a Quick-RNA MiniPrep Kit (Zymo research, CA, USA), followed by reverse transcription to cDNA using iScript cDNA synthesis kit (Bio-Rad, Hercules, CA, USA) and amplified by RT-qPCR in an ABI 7300 system (Applied Biosystems, Waltham, MA, USA). The comparative cycle threshold $(\triangle \triangle \mathrm{CT})$ was used to determine the quantification of gene expression. The gene-level of glyceraldehyde 3-phosphate dehydrogenase (GAPDH) was used for the normalization of tyrosine kinase gene. Primer for tyrosinase and GAPDH are 5'-TGACAAAGCCAAAACCCCCA-3' (forward); 5'-TTGTTCAAAAATACTTCCAGTGTG T-3' (reverse) and 5'-TGTGTCCGTCGRGGATCTGA-3' (forward); 5'-GATGCCTGCTTCACCACCTT3' (reverse).

Cellular tyrosinase activity following FFAR2 gene knockdown. B16F10 melanoma cells were seeded at a density of $5 \times 10^{4}$ cells/well in 24 -well plates. Further, the cells were treated with FFAR2 selective antagonist GLPG0974 $(0.1 \mu \mathrm{M}, \mathrm{GLPG})$ and PA $(4 \mathrm{mM})$ and incubated at $37^{\circ} \mathrm{C}$ in $5 \% \mathrm{CO}_{2}$ for $24 \mathrm{~h}$. Cells were trypsinized and lysed with RIPA buffer (Thermo Fisher Scientific, NJ, USA). The lysed cells were frozen at $-80^{\circ} \mathrm{C}$ and thawed twice followed by centrifugation at $12,000 \mathrm{rpm}$ for $10 \mathrm{~min}$. The supernatant was mixed with $1 \mu \mathrm{g} /$ $\mathrm{mL}$ of Levodopa (L-DOPA, Sigma) in 2:1 ratio in a 96-well plate, incubated at room temperature (RT) for $1 \mathrm{~h}$ and OD at $475 \mathrm{~nm}$ was detected ${ }^{27}$. 
BrdU labeling. B16F10 melanoma cells were grown on 8-well chamber slides (Thermo Fisher Scientific) in DMEM with $10 \%$ FBS, penicillin and streptomycin till $70 \%$ confluency achieved. Bromodeoxyuridine $(10 \mu \mathrm{M}$, BrdU, ACROS, New Jersey, USA) was added to the culture media along with $4 \mathrm{mM}$ PA. BrdU added with PBS in culture media was included as a control. After $24 \mathrm{~h}$, cells were fixed with $4 \%$ perfluoroalkoxy (PFA, Sigma) and then permeabilized with $0.2 \%$ triton X-100 (Sigma) for $10 \mathrm{~min}$. Next, cells were treated with $2 \mathrm{~N} \mathrm{HCl}$ at $37^{\circ} \mathrm{C}$ for $25 \mathrm{~min}$, neutralized with $\mathrm{HCl}$ with $0.1 \mathrm{M}$ Borate buffer $(\mathrm{pH} 8.5)$ for $10 \mathrm{~min}$, and blocked with blocking buffer before incubation anti-BrdU antibody (Abcam, MA, USA) overnight and donkey anti-goat Alexa Fluor 568 IgG $(\mathrm{H}+\mathrm{L})$ (Life technologies) as second antibodies for $1 \mathrm{~h}$ at $4{ }^{\circ} \mathrm{C}$. Nuclei were counterstained with 4,6-diamidino2-phe-nylindole (DAPI, Sigma). Images were acquired with cellSens software (https://www.olympus-lifescience. com.cn/en/software/cellsens/; Version 1.2.1) connected to an Olympus BX63 microscope (Olympus, Tokyo, Japan).

The mouse models created by UV light exposure. Mouse models have been instrumental in advancing the understanding of the many roles of $\mathrm{UVR}^{28}$. UVR increases DOPA-positive melanocytes in the skin, specifically at the site of exposure ${ }^{29}$. The protocol for the treatment of $C$. acnes injection to the mice ears has been described in our previous study ${ }^{3}$ (This reference does not talk about $C$. acnes injection). The ears of ICR mice were injected intradermally with $C$. acnes $\left(10^{7} \mathrm{CFU}\right)$ with and without $2 \%$ PF68 followed by ultraviolet $\mathrm{B}$ (UVB) exposure of $312 \mathrm{~nm}$ wavelength at a dose of $200 \mathrm{~mJ} / \mathrm{cm}^{2}$ using an UV lamp (Model EB-280C, Spectronics Corp., Westbury, NY, USA) for 2 min every day for 3 days. Mouse ears injected with PBS or PF68 followed by UVB exposure were included as a control. In another group of experiment mice, ears were applied with $4 \mathrm{mM}$ PA followed by UV exposure, with PBS as control.

siRNA-Mediated gene silencing of FFAR2. In order to silence FFAR2 gene, we used the chemicallymodified siRNA that targets FFAR2 receptor (FFAR2 siRNA), the siRNA negative control (NC siRNA) and the control without injection (C), which were obtained from GenePharma Co. (Shanghai, China). Their oligonucleotide sequences are siFFAR2: sense strand, 5'-CCGGUGCAGUACAAGUUAUTT-3'; anti-sense strand, 5'-AUAACUUGUACUGCACCGGTT-3'. SiControl: sense strand, 5'-UUCUCCGAACGUGUCACGUTT-3'; anti-sense strand, 5'-ACGUGACACGUUCGGAGAATT-3'. These chemically-modified siRNAs were delivered every day for 3 days by intradermal injection in the ears of mice using a microneedle $(2 \mathrm{mg} / \mathrm{kg}$ of mice weight). From day two, apply with 2\% PA and UV exposure for 3 days. The pretreatment for injecting siRNA to the mouse as described in reference ${ }^{30}$. Then, mice ears were excised and stained on the fourth day.

Western blotting. Mice ears were injected with chemically-modified FFAR2 and control siRNAs followed by application with $2 \%$ PA and UVB exposure for 3 days. On third day mice ears were cut, homogenized and then lysed with RIPA buffer (Thermo Fisher Scientific). Cell lysates (30 $\mu \mathrm{g}$ ) were subjected to $10 \%$ SDS-PAGE gel, which were then transferred to a poly(vinylidene fluoride) (PVDF) membrane (Sigma) and blocked with 5\% $(\mathrm{w} / \mathrm{v})$ nonfat milk before incubation overnight with primary antibodies to FFAR2 Rabbit PolyAb (Proteintech, Rosemont, IL, USA) at $4{ }^{\circ} \mathrm{C}$ or $\beta$-actin (1:1,000; Cusabio Technology, Houston, TX, USA). This was followed by treatment with horseradish peroxidase-conjugated goat anti-rabbit secondary antibody (1:5000) (Thermo Fisher Scientific) for $1 \mathrm{~h}$. Protein bands were detected with a chemiluminescent detection reagent (Thermo Fisher Scientific) and Omega Lum C Imaging System (Gel Co., San Francisco, CA, USA). Protein bands were conducted using ImageJ software (https://imagej.nih.gov/ij/; Version 1.53e).

Melanocyte counting. The cartilages from mouse were removed manually and the skin tissues were soaked in ammonium thiocyanate (Sigma) solution at $37^{\circ} \mathrm{C}$ for $20 \mathrm{~min}$. The epidermal and basal layers were exfoliated from the rest of the skin tissue, and melanocytes were stained by immersing in $0.1 \mathrm{M} \mathrm{PBS}(\mathrm{pH} 7.2)$ containing $0.14 \% \mathrm{~L}-\mathrm{DOPA}$ at RT for $3 \mathrm{~h}$ and the melanocyte count in skin tissues was determined microscopically according to a previously published protocol ${ }^{29}$.

Statistical analyses. Data analysis was performed by unpaired t-test using Prism software (https://www. graphpad.com/; Version 5.01, GraphPad Software, La Jolla, CA, USA). The levels of statistical significance were indicated as the following: ${ }^{\star} P<0.05,{ }^{\star \star} P<0.01,{ }^{* \star} P<0.001$ and $\mathrm{ns}=$ non-significant. The mean \pm standard deviation (SD) for at least three independent experiments except two independent western blotting analysis for Fig. 4C was displayed. Animal experiments were performed with at least three animals per treatment group.

\section{Results}

C. acnes induces fermentation of PF68. Previous studies have demonstrated the fermentation of skin probiotics for induction of SCFA production in the presence of compounds as a carbon source ${ }^{26}$. To investigate if $C$. acnes could ferment PF68, C. acnes was incubated with PF68 in TSB media for 1 day with phenol red as an indicator to the monitor the bacterial fermentation. In TSB media incubated with bacteria alone, the phenol red changed from red to orange due to bacterial replication, whereas in the TSB media containing bacteria and PF68, the phenol red color changed to pale yellow with a decrease in $\mathrm{pH}$ indicating the use of PF68 as a carbon source for fermentation (Fig. 1A). Furthermore, the $\mathrm{OD}_{560}$ of phenol red showed a significant decrease in $\mathrm{pH}$ value in the TSB media containing bacteria and PF68 (Fig. 1B) compared to bacteria or PF68 alone. The SCFAs contained in the C. acnes ferment cultured with PF68 were identified by GC-MS analysis (Fig. 1C) and found to be AA, PA, $\mathrm{BA}$, and I-BA acid, with $\mathrm{PA}^{11}$ having the highest concentration (Fig. 1D). 
A

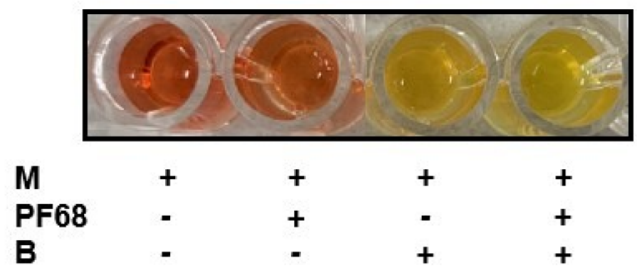

B

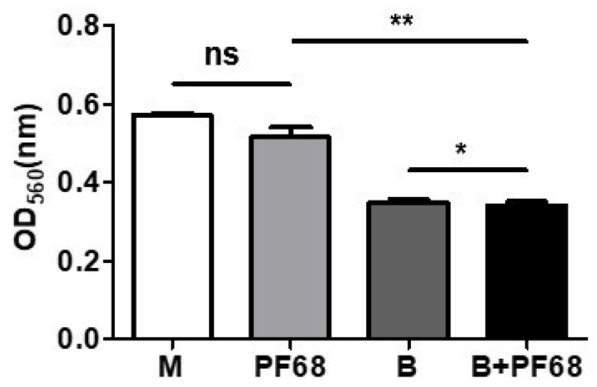

C

AA

PA

BA

I-BA
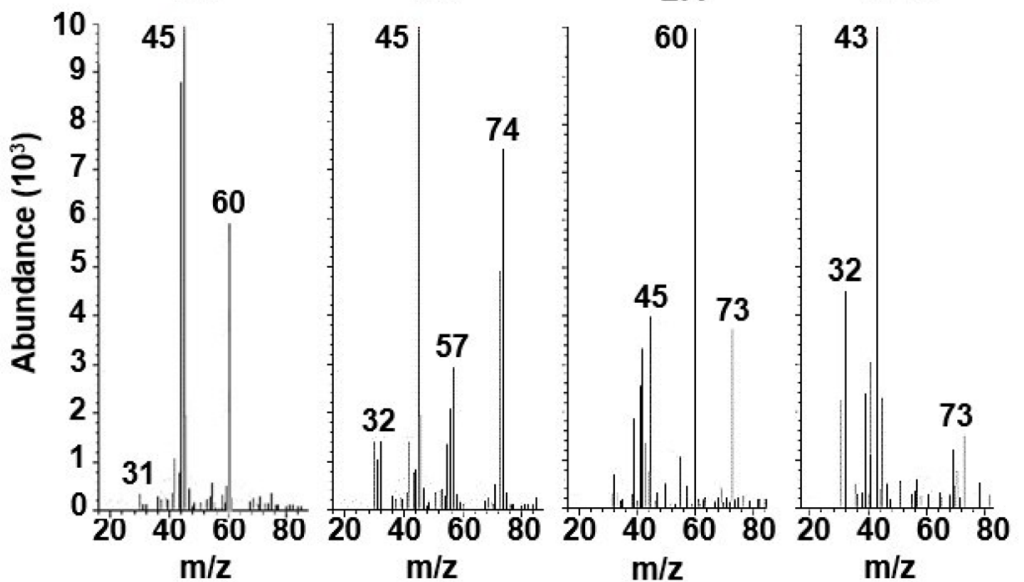

D

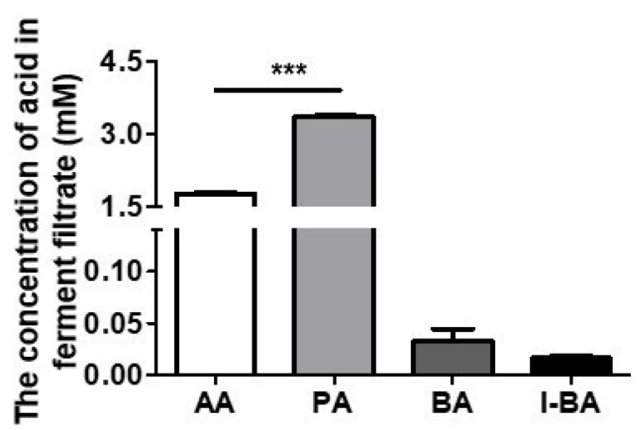

Figure 1. The bacterial fermentation with polymer was accompanied by the decrease in intracellular $\mathrm{pH}$. (A) The color change of phenol red from red to yellow when C. acnes was incubated with/without PF68 in TSB. (B) The prevalence of fermentation was quantified by measuring the optical density of phenol red at $\mathrm{OD}_{560}$. The $\mathrm{pH}$ of media with PF68 and C. acnes was significantly lower than the other groups. (C) The ion chromatogram and mass spectrum showing the SCFAs present in the C. acnes ferment cultured with PF68. (D) Quantification of SCFAs in the $C$. acnes ferment filtrate. A high amount of PA was produced by PF68 fermentation of $C$. acnes. Medium (M), C. acnes (B), acetic acid (AA), propionic acid (PA), butyric acid (BA), and iso-butyric acid (I-BA). Data are the mean \pm SD from three separate experiments. ${ }^{*} P<0.05,{ }^{* *} P<0.01,{ }^{* *} P<0.001$ and ns $=$ nonsignificant (two-tailed t-test).

In vitro effect of PA on melanin production via PA-FFAR2. Fatty acids modulate the degradation of tyrosinase, a crucial enzyme involved in melanin biosynthesis in melanocytes and melanoma cells ${ }^{31}$. Therefore, to detect the in vitro effect of PA on melanin synthesis, B16F10 melanoma cells were treated with $4 \mathrm{mM}$ PA for $48 \mathrm{~h}$, with the decreased expression of the tyrosinase in PA treated melanoma cells compared to the control (Fig. 2A). The immunostaining of PA treated melanoma cells after BrdU labeling showed that PA did not alter the proliferation of melanoma cells (Fig. 2B). Furthermore, the effect of PA and AA, two highly expressed SCFAs in the fermentation filtrate (Fig. 1D) ${ }^{32}$, on melanin production was assessed in melanoma cells, with PA causing 
A

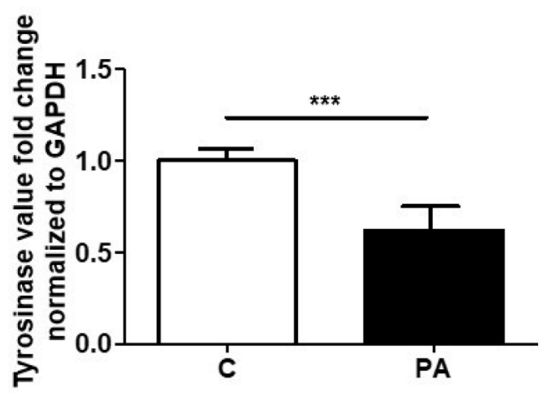

C

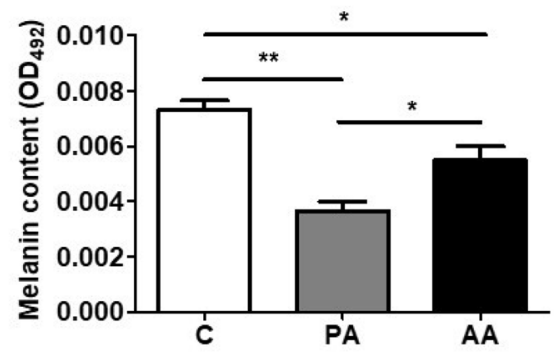

B
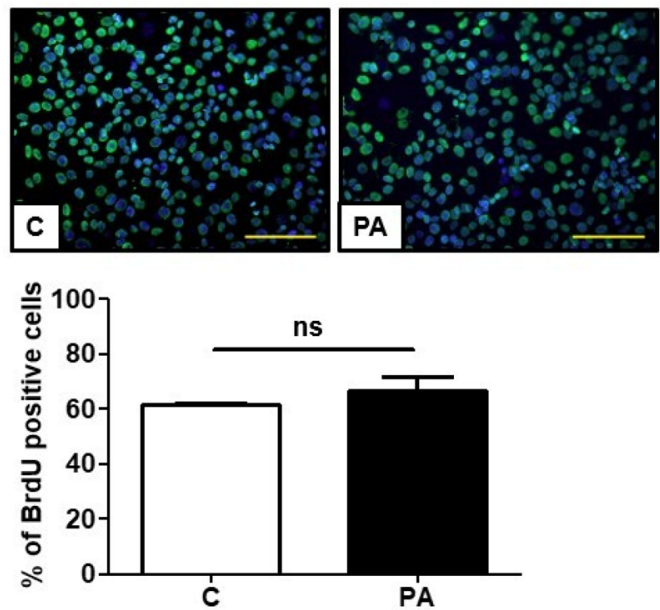

D

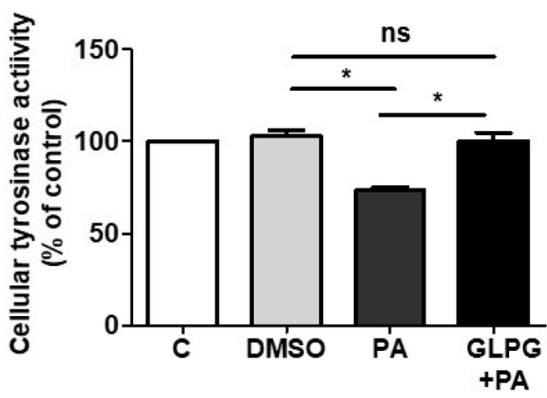

Figure 2. The effects of tyrosinase gene expression and cell proliferation in melanoma cells after PA treatment. (A) Tyrosinase gene expression decreased in PA treated B16F10 melanoma cells quantified by RT-qPCR. (B) The effects of PA on B16F10 melanoma cells evaluated by BrdU labeling. PA treatment did not alter the cell proliferation. (C) Effects of PA and AA in melanocytes. The relatively higher efficacy of PA in attenuating melanin content. (D) Blocking the signaling of FFar2 using GLPG prevents the PA mediated decreased cellular tyrosinase activity in B16F10 cells. Control (C), propionic acid (PA), acetic acid (AA), dimethyl sulfoxide (DMSO), and GLPG0974 (GLPG). Data are the mean \pm SD from three separate experiments. ${ }^{\star} P<0.05,{ }^{* *} P<0.01$ and ns =non-significant (two-tailed t-test). Scale bar $=120 \mu \mathrm{m}$.

a $>$ twofold decrease in melanin production compared to AA (Fig. 2C). SCFAs, like PA and AA, regulate their functions via interaction with FFAR2 and FFAR3, with PA being among the most potent SCFA for both these receptors $^{33}$. Considering PA regulation via its interaction with FFAR2, blocking the signaling of this receptor using a selective inhibitor could be a useful approach to evaluate the role of PA in the regulation of melanogenesis. The cellular tyrosinase activity was unchanged by blocking FFAR2 using the FFAR2 selective antagonist GLPG before treatment with PA and decreased without GLPG in contrast to the control group (Fig. 2D). These results demonstrated the effective role of PA-FFAR2 in the attenuation of the melanin content by inhibiting tyrosinase kinase activity in melanoma cells with unaltered cell proliferation.

The mixture of $C$. acnes and PF68 inhibited UVB-induced functioning melanocyte in mouse ear. The topical application of fermentation extracts or fatty acids has been shown to decrease UV-induced skin hyperpigmentation by regulating tyrosinase degradation ${ }^{34,35}$. Previous studies demonstrated that melanogenesis after UV exposure is due to the activation in functioning melanocytes in the epidermis or dermis ${ }^{36}$. Having established that PA as a major SCFA from C. acnes fermentation of PF68 could effectively reduce melanin production in vitro, we then examined the effect of $C$. acnes plus PF68 on it in vivo. The ears of ICR mice were exposed to UVB every other day for 3 days concurrent with the injection of $C$. acnes and PF68. Injection with PBS or C. acnes or PF68 alone were included as controls. The epidermal and basal layers were exfoliated from the skin tissue in the mouse ear and the melanocytes were stained with L-DOPA. There was a significant increase of the melanocytes number after UVB exposure, with no change after injection with C. acnes or PF68 alone. However, a significant reduction in number of DOPA-positive melanocytes was detected in the UVB exposure groups after treatment with a mixture of C. acnes and PF68 (Fig. 3A). 
A
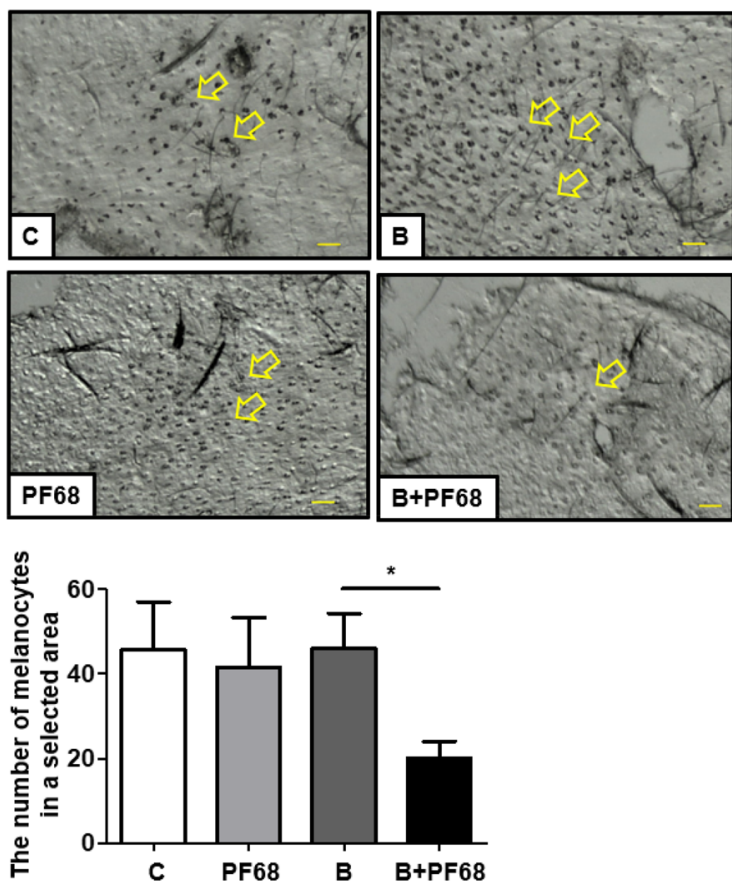

B
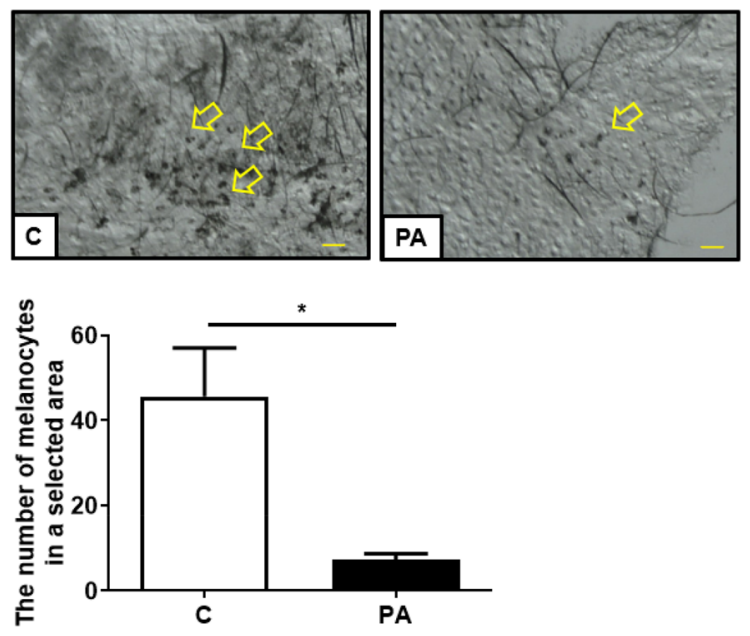

C

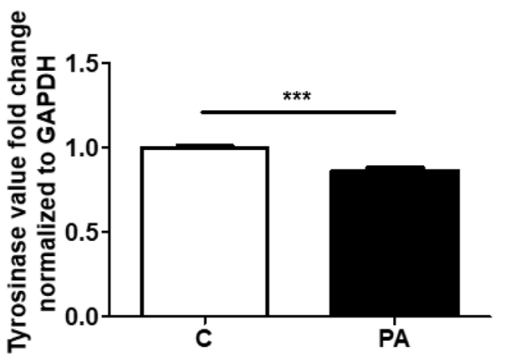

Figure 3. Induction of melanocytes by UVB and inhibition by different treatments. (A) The ears of mice were applied PF68 + C. acnes. The same amount of PBS, PF68 only, and C. acnes only were applied as control. Specimens were examined microscopically for the effects of PF68 +C. acnes treatment on the DOPA-positive melanocytes proliferation in the epidermis. (B) The ears of mice were applied PA. The same amount of PBS was applied as control. Specimens were examined microscopically for the effects of PA treatment on the DOPA-positive melanocytes proliferation in the epidermis. (C) Tyrosinase gene expression after PA treatment in vivo was quantified by RT-qPCR. Control (C), C. acnes (B), propionic acid (PA). The arrowhead in A and B shows the staining of DOPA-positive melanocytes that was counted in the same millimeter squared. Data are the mean \pm SD from three separate experiments was replayed. ${ }^{\star} P<0.05,{ }^{* *} P<0.001$ (two-tailed t-test). Scale bar $=60 \mu \mathrm{m}$.

Amelioration of UVB-induced functioning melanocyte in mouse ear by PA, a C. acnes fermentation metabolite. The effect of the direct application of PA on UV-induced melanogenesis was assessed. UV-induced hyperpigmentation and tyrosinase expression in mouse ear skin in the control group was significantly lowered after the topical application of PA for 3 days (Fig. 3B). Thus, PA, a metabolite from PF68 fermentation of $C$. acnes inhibits functioning melanocytes production with melanin synthesis by UVB-induced. Tyrosinase expression was noticeably decreased in PA treated cells compared to the control (Fig. 3C).

PA inhibits UVB-induced functioning melanocytes via FFAR2 in vivo. To determine if the reduction in exogenous melanogenesis occurred via PA interaction with its cognate receptor, FFAR2 was knocked down in the skin melanocytes of the mouse ear, followed by UVB exposure and PA treatment. UVB-induced an increase in the proliferation of melanocytes and tyrosinase activity was significantly reduced in the mouse ear epidermis injected with NC siRNA after application of PA (Fig. 4A,B). However, the number of melanocytes and tyrosinase activity remains unchanged even after PA application in UVB irradiated mouse ear epidermis knocked down with FFAR2. We confirmed the FFAR2 gene knockdown by measuring the protein expression level of FFAR2 by Western blot analysis (Fig. 4C). Thus, PA interferes with melanogenesis by suppressing the activity of tyrosinase, in which PA-FFAR2 plays a potential role in melanin production.

\section{Discussion}

Melanin, a critical factor of skin defense against UV-irradiation, is synthesized in the melanosomes of melanocytes, transferred to neighboring keratinocytes via the dendritic tips, and eventually distributed throughout the skin epidermis ${ }^{37}$. Bacteria fermentation metabolites are increasingly used in skin therapy due to their beneficial effect on UV-induced skin inflammation and infectious disorders ${ }^{3}$. In this study, we demonstrated that PA, the 
A
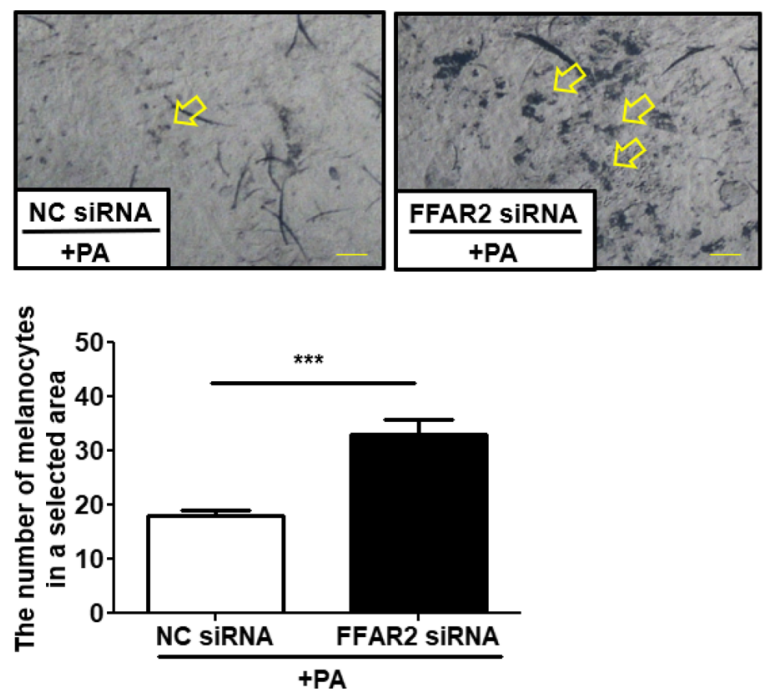

B
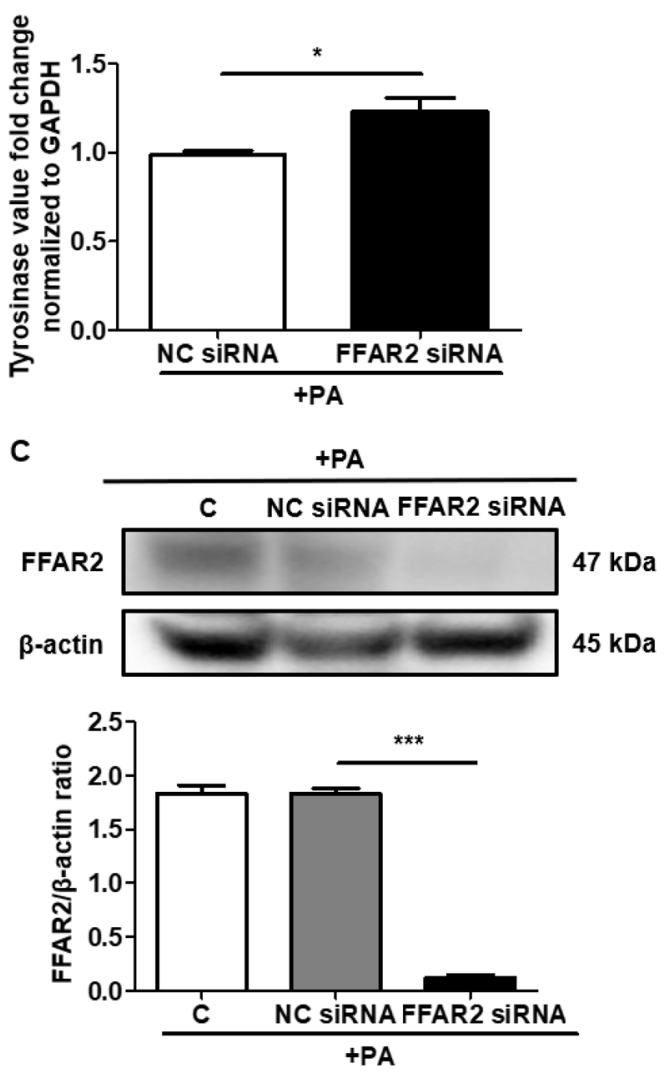

Figure 4. The siRNA knockdown efficiency of the FFAR2 gene, along with the topical application of PA and UV exposure to ICR mice ears. (A) Specimens were examined microscopically for the effects of PA treatment after FFAR2 gene knockdown on the DOPA-positive melanocytes proliferation in the epidermis. (B) Tyrosinase gene expression in vivo quantified by RT-qPCR. (C) Western blotting to confirm FFAR2 gene knockdown and the expression of FFAR2 and $\beta$-actin in mice ears. Full-length gels images are available in Supplemental Information. Control (C), propionic acid (PA), negative control (NC). The arrowhead in A and B shows the staining of DOPA-positive melanocytes that was counted in the same millimeter squared. Data A and B are the mean \pm SD from three separate experiments except two independent western blotting analysis for $\mathrm{C}$ was replayed. ${ }^{\star} P<0.05,{ }^{* *} P<0.001$ (two-tailed t-test). Scale bar $=60 \mu \mathrm{m}$.

major metabolite from PF68 fermentation of C. acnes, reduced UVB-induced functioning melanocyte levels by inhibiting tyrosinase activity in vitro and in vivo via FFAR2.

Previous studies demonstrated the inhibition of melanogenesis through tyrosinase inhibition by fermentation metabolites from probiotic LA and Lactobacillus paracasei bacteria ${ }^{24,38,39}$. Also, the high molecular weight PEGbased polymer $(\sim 20,000)$ was completely degraded through fermentation by gram-negative bacteria producing acetate and propionate ${ }^{40}$. In this study, PA $(\sim 4 \mathrm{mM})$ was the most abundant SCFA in the filtrate from PF68 fermentation of $C$. acnes and reduced the melanin content in melanocytes more effectively than AA. Furthermore, treatment with $4 \mathrm{mM}$ PA significantly inhibited cellular tyrosinase activity in melanocytes proving that inhibition of melanogenesis by PA occurred via the reduction of tyrosinase gene expression. People have the same number of melanocytes that is not the reason to affect skin pigmentation but the activation in functioning melanocytes by UV-irradiation ${ }^{36,41}$. Here, $4 \mathrm{mM}$ PA treatment did not alter melanocyte proliferation, indicating that it is an effective treatment option, causing no cellular damage. The reduced melanocytes number and tyrosinase activity were also observed in mice ear skin tissue injected with a mixture of C. acnes and PF68, demonstrating that the inhibition of melanogenesis is likely to be mediated through fermentation metabolites from C. acnes fermentation using PF68 as a carbon source. Additionally, our study validated PA as an excellent antimicrobial agent, with no alteration in the growth of its parent bacteria C. acnes, showing PA as a potent metabolite that does not disrupt the balance of the skin microbiome ${ }^{11}$.

Melanin production is initiated and regulated by several signaling systems, whereby tyrosinase catalyzes the conversion of tyrosine to DOPA, and into dopaquinone, leading to the formation of melanocytic pigments ${ }^{42,43}$. In most cases, skin lightening reagents act at various levels of melanin production via FFAR2 to affect tyrosinase activity or directly targeting tyrosinase to inhibit melanogenesis in the skin ${ }^{44-46}$. SCFAs such as PA and AA exert their effects through binding to their selective cognate receptors like FFAR2 or FFAR3, with PA being among the most potent SCFA for both receptors ${ }^{47}$. GLPG, a selective FFAR2 antagonist, and siRNA-mediated gene silencing of FFAR2 to support the blocking of FFAR2 $2^{3,48}$. In the current study, the increased melanocytes 
number and tyrosinase gene expression in mice ear skin tissue did not changed in response to UVB radiation even after PA application to FFAR2 knockdown in mice (Fig. 4A). Furthermore, tyrosinase gene expression was decreased after PA treatment in vitro, however, blocking the FFAR2 gene with GLPG, a selective FFAR2 antagonist, and ameliorated the effect of PA to attenuate tyrosinase gene expression. After blocking FFAR2 as loss of FFAR2 results in enhanced expression of tyrosinase activity after PA treatment. Although both PA and AA, the fermentation metabolites from C. acnes, have a similar affinity for FFAR2 binding ${ }^{20}$, the relatively lower efficacy of AA in attenuating tyrosinase activity in melanocytes validates the therapeutic potential of PA as a postbiotic against melanogenesis.

The photo damaging effects caused by UVR on the skin have attracted extensive attention. Sun screening and anti-hyperpigmentation products are widely used but their safety, skin penetration, and therapeutic efficacy are still in question ${ }^{49}$. Although avobenzone is a common component in sunscreens owing to its high efficacy against UV, it is photo unstable and therefore not safe, and has been detected in blood after chronic applications $\mathrm{s}^{50,51}$. Development of effective skin whitening through blocking tyrosinase expression by fermentation metabolites from probiotic bacteria or polymers as a carbon source is an effective and natural way to suppress melanogenesis ${ }^{38,39}$. As the use of live probiotics for cosmetics is strictly regulated, the promotion of their beneficial effects through fermentated metabolites from live probiotic bacteria has become a feasible application. Besides, PF68 as a prebiotic can boost the production of SCFAs from C. acnes fermentation and has been used to enhance the biological stability and assist various therapeutic agents such as 6-mercaptopurine loaded microspheres in their interaction with the human body ${ }^{52}$. Furthermore, PF68 can be incorporated into cell membranes and translocate into cells and has been used as a stable gel carrier for antimicrobial agents, increasing the surface solubility of the drug in skin treatment ${ }^{12,23}$. Hence, PF68 is considered a safe and effective options for the development of pharmaceuticals and cosmetics. In addition to the function of PF68 as a fermentation initiator to augment the fermentation activity of $C$. acnes, it also has the potential to be an adjuvant to reduce the effective doses of medicine reagents and improve the solubility of poor-water soluble drugs.

Overall, these results demonstrate that the PA-FFAR2 interaction may be a central mechanism in the effect of probiotics or postbiotics on hyperpigmentation caused by UVR. PA treatment did not affect melanocyte proliferation. Compared with chemical therapies, the metabolites of probiotics are milder and natural ${ }^{53}$. Although the exact mechanism by which the metabolites of PF68 fermentation of C. acnes inhibit melanogenesis is unclear, the evidence from the present study suggests the involvement of the SCFAs-FFAR2-tyrosinase pathway. These results are beneficial for the future clinical treatment of pigmentation disorders and to develop cosmetics that increases the range of applications of whitening products. Lastly, the diversity of probiotics offers personalized treatments for hyperpigmentation and the development of dedicated products with higher performance.

Received: 21 February 2021; Accepted: 18 May 2021

Published online: 07 June 2021

\section{References}

1. Patra, V., Byrne, S. N. \& Wolf, P. The skin microbiome: is it affected by uv-induced immune suppression?. Front Microbiol. 7, 1235. https://doi.org/10.3389/fmicb.2016.01235 (2016).

2. Wang, Y. et al. The response of human skin commensal bacteria as a reflection of UV radiation: UV-B decreases porphyrin production. PLoS ONE. https://doi.org/10.1371/journal.pone.0047798 (2012).

3. Keshari, S. et al. Butyric acid from probiotic staphylococcus epidermidis in the skin microbiome down-regulates the ultravioletinduced pro-inflammatory IL-6 cytokine via short-chain fatty acid receptor. Int. J. Mol. Sci. https://doi.org/10.3390/ijms201844 77 (2019).

4. Hawryluk, E. B., Oztan, A. \& Fisher, D. E. Effects of ultraviolet exposure behaviors on skin pigmentation and melanoma. J. Pigm. Disord. https://doi.org/10.4172/jpd.1000113 (2014).

5. Tu, C. X. et al. Curcumin inhibits melanogenesis in human melanocytes. Phytother Res 26, 174-179. https://doi.org/10.1002/ptr. 3517 (2012).

6. Roth, R. R. \& James, W. D. Microbial ecology of the skin. Annual Rev. Microbiol. 42, 441-464 (1988).

7. Grice, E. A. \& Segre, J. A. The skin microbiome. Nat Rev Microbiol 9, 244-253. https://doi.org/10.1038/nrmicro2537 (2011).

8. Ji, G., Beavis, R. \& Novick, R. P. Bacterial interference caused by autoinducing peptide variants. Science 276, 2027-2030. https:// doi.org/10.1126/science.276.5321.2027 (1997).

9. Kober, M. M. \& Bowe, W. P. The effect of probiotics on immune regulation, acne, and photoaging. Int. J. Womens Dermatol. 1, 85-89. https://doi.org/10.1016/j.ijwd.2015.02.001 (2015).

10. Baur, M., Breton, L., Couzy, F. \& Gueniche, A. Use of probiotic lactic acid bacteria for preventing ultraviolet radiation induced inflammatory or allergic reaction or immunosuppression in the skin. French patent (2002).

11. Shu, M. et al. Fermentation of Propionibacterium acnes, a commensal bacterium in the human skin microbiome, as skin probiotics against methicillin-resistant Staphylococcus aureus. PLOS ONE. https://doi.org/10.1371/journal.pone.0055380 (2013).

12. Futsaether, C. M., Kjeldstad, B. \& Johnsson, A. Intracellular $\mathrm{pH}$ changes induced in Propionibacterium acnes by UVA radiation and blue light. J. Photochem. Photobiol., B 31, 125-131 (1995).

13. Kjeldstad, B. Photoinactivation of propionibacterium acnes by near-ultraviolet light. Z Naturforsch C. 39, 300-302 (1984).

14. Woolridge, J. Galactomyces ferment filtrate reduces melanin synthesis and oxidative stress in normal human melanocytes. J. Am. Acad. Dermatol. https://doi.org/10.1016/j.jaad.2014.01.527 (2014).

15. Ando, H., Funasaka, Y. \& Oka, M. Possible involvement of proteolytic degradation of tyrosinase in the regulatory effect of fatty acids on melanogenesis. J. Lipid Res. 40, 1312-1316 (1990).

16. D, D. Z. Skin lightening preparations and the hydroquinone controversy. Dermatol. Therapy 20, 308-313 (2007).

17. R., K. Fairness via formulations: A review of cosmetic skin-lightening ingredients. J. Cosmet. Sci. 63, 43-54 (2012).

18. Read, J. E. et al. Dynamic changes in gene expression and signalling during trophoblast development in the horse. Reproduction https://doi.org/10.1530/rep-18-0270 (2018)

19. Pan, P. et al. Loss of FFAR2 promotes colon cancer by epigenetic dysregulation of inflammation suppressors. Int J Cancer 143, 886-896. https://doi.org/10.1002/ijc.31366 (2018).

20. Hong, Y. H. et al. Acetate and propionate short chain fatty acids stimulate adipogenesis via GPCR43. Endocrinology 146, 5092-5099. https://doi.org/10.1210/en.2005-0545 (2005). 
21. Le Poul, E. et al. Functional characterization of human receptors for short chain fatty acids and their role in polymorphonuclear cell activation. J. Biol. Chem. 278, 25481-25489. https://doi.org/10.1074/jbc.M301403200 (2003).

22. Wang, Y. et al. Propionic acid and its esterified derivative suppress the growth of methicillin-resistant Staphylococcus aureus USA300. Benef Microbes 5, 161-168. https://doi.org/10.3920/BM2013.0031 (2014).

23. Bridgett, M. J. \& Davies, M. Control of staphylococcal adhesion to polystyrene surfaces by polymer surface modification with surfactants. Biomaterials 13, 411-416 (1992).

24. Kao, M. S. et al. Microbiome precision editing: Using PEG as a selective fermentation initiator against methicillin-resistant Staphylococcus aureus. Biotechnol J. https://doi.org/10.1002/biot.201600399 (2017).

25. Treter, J. et al. Washing-resistant surfactant coated surface is able to inhibit pathogenic bacteria adhesion. Appl. Surf. Sci. 303, 147-154. https://doi.org/10.1016/j.apsusc.2014.02.123 (2014).

26. Keshari, S. et al. Skin cutibacterium acnes mediates fermentation to suppress the calcium phosphate-induced itching: a butyric acid derivative with potential for uremic pruritus. J. Clin. Med. https://doi.org/10.3390/jcm9020312 (2020).

27. Ko, H. H. et al. Eupafolin, a skin whitening flavonoid isolated from Phyla nodiflora, downregulated melanogenesis: Role of MAPK and Akt pathways. J. Ethnopharmacol. 151, 386-393. https://doi.org/10.1016/j.jep.2013.10.054 (2014).

28. Day, C. P., Marchalik, R., Merlino, G. \& Michael, H. Mouse models of UV-induced melanoma: genetics, pathology, and clinical relevance. Lab Invest. 97, 698-705. https://doi.org/10.1038/labinvest.2016.155 (2017).

29. Hiramoto, K., Yanagihara, N., Sato, E. F. \& Inoue, M. Ultraviolet B irradiation of the eye activates a nitric oxide-dependent hypothalamopituitary proopiomelanocortin pathway and modulates functions of alpha-melanocyte-stimulating hormone-responsive cells. J Invest. Dermatol. 120, 123-127. https://doi.org/10.1046/j.1523-1747.2003.12004.x (2003).

30. Pan, J. et al. Intradermal delivery of STAT3 siRNA to treat melanoma via dissolving microneedles. Sci. Rep. 8, 1117. https://doi. org/10.1038/s41598-018-19463-2 (2018).

31. Ando, H. et al. Fatty acids regulate pigmentation via proteasomal degradation of tyrosinase: A new aspect of ubiquitin-proteasome function. J. Biol. Chem. 279, 15427-15433. https://doi.org/10.1074/jbc.M313701200 (2004).

32. LeBlanc, J. G. et al. Beneficial effects on host energy metabolism of short-chain fatty acids and vitamins produced by commensal and probiotic bacteria. Microb. Cell Fact 16, 79. https://doi.org/10.1186/s12934-017-0691-z (2017).

33. Ulven, T. Short-chain free fatty acid receptors FFA2/GPR43 and FFA3/GPR41 as new potential therapeutic targets. Front Endocrinol (Lausanne) 3, 111. https://doi.org/10.3389/fendo.2012.00111 (2012).

34. Ahn, H. Y., Choo, Y. M. \& Cho, Y. S. Anti-pigmentation effects of eight phellinus linteus-fermented traditional crude herbal extracts on brown guinea pigs of ultraviolet b-induced hyperpigmentation. J Microbiol Biotechnol 28, 375-380. https://doi.org/10.4014/ jmb.1711.11043 (2018).

35. Shigeta, Y. et al. Skin whitening effect of linoleic acid is enhanced by liposomal formulations. Biol. Pharm. Bull. 27, 591-594 (2004).

36. Jimbow, K. \& Uesugi, T. New melanogenesis and photobiological processes in activation and proliferation of precursor melanocytes after UV-exposure: Ultrastructural differentiation of precursor melanocytes from Langerhans cells. J. Invest. Dermatol. 78, 108-115. https://doi.org/10.1111/1523-1747.ep12505758 (1982).

37. Schallreuter, K. U., Kothari, S., Chavan, B. \& Spencer, J. D. Regulation of melanogenesis-controversies and new concepts. Exp. Dermatol. 17, 395-404. https://doi.org/10.1111/j.1600-0625.2007.00675.x (2008).

38. Chen, Y. M., Shih, T. W., Chiu, C. P., Pan, T.-M. \& Tsai, T.-Y. Effects of lactic acid bacteria-fermented soy milk on melanogenesis in B16F0 melanocytes. J. Funct. Foods 5, 395-405. https://doi.org/10.1016/j.jff.2012.11.012 (2013).

39. Bs, N. Y. K., Ms, H. S. K. \& Lee, H. Y. Effect of inhibition on tyrosinase and melanogenesis of Agastache. J. Cosmetic Dermatol. 16, 407-415. https://doi.org/10.1111/jocd.12264 (2017).

40. Bernhard, S. \& Marion, S. Fermentative degradation of polyethylene glycol by a strictly anaerobic, gram-negative, nonsporeforming bacterium, Pelobacter venetianus sp. nov. Appl. Environ. Microbiol. 45, 1905-1913 (1983).

41. Kittles, R. Nature, origin, and variation of human pigmentation. J. Black Stud. (1995).

42. D’Mello, S. A., Finlay, G. J., Baguley, B. C. \& Askarian-Amiri, M. E. Signaling Pathways in Melanogenesis. Int. J. Mol. Sci. https:// doi.org/10.3390/ijms17071144 (2016).

43. Seim, I., Ma, S. \& Gladyshev, V. N. Tyrosinase gene expression in human tissues. Pigment Cell Res. 6, 400-405. https://doi.org/10. 1038/npjamd.2016.14 (1993).

44. Kumar, K. J. et al. In vitro and in vivo studies disclosed the depigmenting effects of gallic acid: A novel skin lightening agent for hyperpigmentary skin diseases. BioFactors 39, 259-270. https://doi.org/10.1002/biof.1064 (2013).

45. Truong, X. T. et al. Protocatechuic acid from pear inhibits melanogenesis in melanoma cells. Int. J. Mol. Sci. https://doi.org/10. 3390/ijms18081809 (2017).

46. Oliveira, K. B., Palú, É., Weffort-Santos, A. M. \& Oliveira, B. H. Influence of rosmarinic acid and Salvia officinalis extracts on melanogenesis of B16F10 cells. Rev. Bras 23, 249-258. https://doi.org/10.1590/s0102-695x2012005000135 (2013).

47. Sawzdargo, M. et al. A cluster of four novel human $\mathrm{G}$ protein-coupled receptor genes occurring in close proximity to CD22 gene on chromosome 19q13.1. Biochem Biophys Res Commun 239, 543-547. https://doi.org/10.1006/bbrc.1997.7513 (1997).

48. Negari, I. P., Keshari, S. \& Huang, C. M. Probiotic activity of Staphylococcus epidermidis induces collagen type I production through FFaR2/p-ERK Signaling. Int J Mol Sci 22. https://doi.org/10.3390/ijms22031414 (2021).

49. Rai R, Shanmuga SC \& C., S. Update on photoprotection. Update on photoprotection. Indian J Dermatol. 57, 335-342 (2012).

50. Kullavanijaya P \& HW., L. Photoprotection. J Am Acad Dermatol. 52, 937-958 (2005).

51. Matta, M. K., Zusterzeel, R. \& Pilli, N. R. Effect of sunscreen application under maximal use conditions on plasma concentration of sunscreen active ingredients. JAMA 321, 2082-2091 (2019).

52. Praveen B. Kajjari, Lata S. Manjeshwar \& Aminabhavi, T. M. Novel Blend Microspheres of Poly(3-hydroxybutyrate) and Pluronic F68/127 for Controlled Release of 6-Mercaptopurine. J. APPL. POLYM. SCI. 131 (2014).

53. Shenderov, B. A. Metabiotics: novel idea or natural development of probiotic conception. Microb Ecol Health Dis 24, doi:https:// doi.org/10.3402/mehd.v24i0.20399 (2013).

\section{Acknowledgements}

The authors thank Dr. Cheng Ching-Yi for the technical support of this project. We would also like to thank Dr. Lan Tian for providing valuable comments and proofreading.

\section{Author contributions}

C.-M.H.: Conceptualization; H.-J.K., and Y.-H.W.: methodology; S.K.: validation; H.-J.K., S.K., and Y.-H.W.: formal analysis; H.-J.K.: investigation; C.-M.H.: resources; H.-J.K., and C.-M.H.: data curation; H.-J.K.: writingoriginal draft preparation; S.K., J.J.Y., S.S., C.-C.C., and C.-M.H.: writing-review and editing; C.-M.H.: supervision, project administration, funding acquisition. All authors have read and agreed to the published version of the manuscript. 


\section{Funding}

This work was funded by 106/107/108-Landseed Hospital-NCU joint grants and Ministry of Science and Technology (MOST) Grants 108-2622-B-008-001-CC1; 108-2314-B-008-003-MY3, and 107-2923-B-008-001-MY3.

\section{Competing interests}

The authors declare no conflict of interests.

\section{Additional information}

Supplementary Information The online version contains supplementary material available at https://doi.org/ 10.1038/s41598-021-91386-x.

Correspondence and requests for materials should be addressed to C.-M.H.

Reprints and permissions information is available at www.nature.com/reprints.

Publisher's note Springer Nature remains neutral with regard to jurisdictional claims in published maps and institutional affiliations.

(c) (i) Open Access This article is licensed under a Creative Commons Attribution 4.0 International License, which permits use, sharing, adaptation, distribution and reproduction in any medium or format, as long as you give appropriate credit to the original author(s) and the source, provide a link to the Creative Commons licence, and indicate if changes were made. The images or other third party material in this article are included in the article's Creative Commons licence, unless indicated otherwise in a credit line to the material. If material is not included in the article's Creative Commons licence and your intended use is not permitted by statutory regulation or exceeds the permitted use, you will need to obtain permission directly from the copyright holder. To view a copy of this licence, visit http://creativecommons.org/licenses/by/4.0/.

(C) The Author(s) 2021 